\title{
A critical assessment of the in-vitro measurement of cortical bone stiffness with ultrasound
}

\author{
L. Peralta ${ }^{\mathrm{a}}$, X. Cai ${ }^{\mathrm{a}, *}$, P. Laugier ${ }^{\mathrm{a}}$, Q. Grimal ${ }^{\mathrm{a}}$ \\ ${ }^{a}$ Sorbonne Universités, UPMC Univ Paris 06, INSERM UMR-S 1146, CNRS UMR 7371, Laboratoire d'Imagerie \\ Biomédicale, 75006 Paris, France
}

\begin{abstract}
Elasticity assessment based on bulk wave velocity (BWV) measurements is the most popular technique to characterize the anisotropic stiffness tensor in cortical bone. Typically, a cuboid bone specimen is cut with its sides along the different anatomical directions. Then, the velocity of shear and longitudinal waves propagating along different directions are assessed, from which stiffness coefficients are calculated. Despite the importance of obtaining accurate elasticity values for bone research, there is no generally accepted protocol to measure BWV and the precision of the technique has been seldom investigated. The purpose of this work is to critically assess the method to measure BWV on cuboid specimens in terms of ultrasound frequency, specimen size and signal processing technique. In this study, we measured polycarbonate specimens of different dimensions and 55 human bone specimens with different transducers using frequencies ranging from 2.25 to $10 \mathrm{MHz}$ and 1 to $5 \mathrm{MHz}$ for longitudinal and shear waves, respectively. We compared four signal processing methods to detect the wave arrival time. The main results are that, (1) the measurement of shear waves is more complex than that of longitudinal wave, being less precise and more sensitive to sample size; (2) the estimated stiffness depends on the signal processing technique used (up to $10 \%$ variation for shear coefficients of bone); and (3) bone stiffness assessed from BWV using the first arrival of the signal to determine the time-of-flight is not different from stiffness assessed using resonant ultrasound spectroscopy (RUS). These results evidence that the measurement method can have an effect on the stiffness values estimates and hence, a well-defined protocol is needed to accurately measure bone stiffness coefficients based on BWV.
\end{abstract}

Keywords: ultrasound velocity, cortical bone, resonant ultrasound spectroscopy

PACS: 71.35.-y, 71.35.Lk, 71.36.+c

\section{Introduction}

Measurement of bone elastic properties and the understanding of their variations are a key in elucidating the mechanical effects of skeletal pathologies such as osteoporosis. Also, elastic properties are necessary inputs of finite element models of the skeleton for the accurate computation of stresses and strains (van Rietbergen and Ito, 2015; Engelke et al., 2016).

According to the Christoffel equation, the coefficients of the stiffness tensor of an anisotropic elastic solid can be deduced from the phase velocity of shear and longitudinal ultrasound (US)

\footnotetext{
* Corresponding author

Email address: xiran.cai@upmc.fr (X. Cai)
}

bulk waves propagating along different material directions. Most of the knowledge on the stiffness of cortical bone, which is an orthotropic or transverse isotropic material (Ashman et al., 1984; Rho, 1996; Orías et al., 2009; Rudy et al., 2011) has been obtained through the measurement of ultrasonic bulk wave velocity (BWV) ex vivo along different anatomical directions using cuboid specimens. Since the method was introduced to measure bone in the 1960's (Lang, 1969), and until recently, this technique has been applied to assess bone elasticity and relate it to age (Lefèvre et al., 2015), anatomical location (Orías et al., 2009; Schwartz-Dabney and Dechow, 2002) or others bone properties such as microstructure (Granke et al., 2011) and extravascular bone tissue properties (Baumann et al., 
2012).

However, in bone studies such as those cited above, the phase velocity is not measured but rather a signal velocity that is used in place of phase velocity to determine the stiffness coefficients from Christoffel equation. The interpretation of this signal velocity is complicated by the modifications of the pulse shape during propagation due to frequency-dependent attenuation, dispersion and possible interference effects related to reflections within the specimen (Haiat et al., 2006; Kohles et al., 1997). In practice the signal velocity is obtained by determining the first deviation from zero (Njeh et al., 1996), one zero-crossing (Haiat et al., 2006) or a thresholding (Nicholson et al., 1996). In general, these different markers yield different ultrasound velocity values (Haiat et al., 2006).

The possible biases of the method of measurement of BWV for the estimation of cortical bone stiffness have not been thoroughly discussed to the best of our knowledge. It appears that there is no generally accepted protocol to measure BWV in cortical bone in terms of US frequency (frequencies in the range 1 to $20 \mathrm{MHz}$ have been used), signal processing technique and specimen size (specimens of characteristic size between 0.5 and $10 \mathrm{~mm}$ have been used) (Lang, 1969; Kohles et al., 1997; Ashman et al., 1984; Lefèvre et al., 2015). Furthermore, the signal processing method used to estimate the velocity is rarely specified in the studies.

The aim of this study is to elucidate the effects of the signal processing to measure BWV on cuboid specimens of cortical bone, discuss the choice of transducer frequency and the possible influence of specimen size. Based on BWV measurements of polycarbonate specimens of different sizes and bone specimens, and comparing with stiffness estimated by resonant ultrasound spectroscopy (RUS), we propose guidelines to standardize the BWV measurement of cortical bone stiffness. Section 2 presents the equations used to assess the stiffness tensor from BWV measurements. The methodology is described in Section 3, which contains four subsections. The samples included in this study are described in Subsection 3.1. Subsection 3.2 presents the ultrasonic velocity measurements and the RUS method is outlined in Subsection 3.3. Statistics are presented in Subsection 3.4. Finally, results are illustrated and discussed in Sections 4 and 5 , respectively and concluding remarks end the paper in Section 6.

\section{Theory}

The stiffness tensor of an orthotropic material has nine independent coefficients. The tensor writes in matrix form using Voigt notation as,

$$
C_{i j}=\left(\begin{array}{cccccc}
C_{11} & C_{12} & C_{13} & 0 & 0 & 0 \\
C_{12} & C_{22} & C_{23} & 0 & 0 & 0 \\
C_{13} & C_{23} & C_{33} & 0 & 0 & 0 \\
0 & 0 & 0 & C_{44} & 0 & 0 \\
0 & 0 & 0 & 0 & C_{55} & 0 \\
0 & 0 & 0 & 0 & 0 & C_{66}
\end{array}\right)
$$

According to the Christoffel equation (Auld, 1973), the 6 diagonal terms can be determined by propagating longitudinal and shear waves along the principal material axes $(1,2,3)$,

$$
\begin{aligned}
C_{i i} & =\rho v_{i i}^{2}(i=1,2,3) \\
C_{44} & =\rho v_{23}^{2}=\rho v_{32}^{2} \\
C_{55} & =\rho v_{13}^{2}=\rho v_{31}^{2} \\
C_{66} & =\rho v_{12}^{2}=\rho v_{21}^{2}
\end{aligned}
$$

where $\rho$ is the mass density, $v_{i i}$ is the velocity of a longitudinal wave propagating in direction $i$, and $v_{i j}$ is the velocity of a shear wave propagating in direction $i$ with particle motion in direction $j$. The off-diagonal terms of the stiffness tensor can be obtained by measuring BWV in directions at a 45 angle from the material axes. However this has rarely been done to measure cortical bone due to the limited size of the samples.

If the material is isotropic, then, its stiffness tensor can be characterized by two constants : $C_{11}=$ $C_{22}=C_{33}$ and $C_{44}=C_{55}=C_{66}$.

\section{Method}

\subsection{Specimens}

Eleven polycarbonate (PC) specimens were prepared to investigate the effect of specimen size on BWV. PC was chosen because its acoustic properties (BWV, intrinsic attenuation) are close to those of cortical bone (Phillips et al., 1977; Lakes et al., 1986). The density of the PC was $\rho=1.187 \mathrm{~g} / \mathrm{cm}^{3}$. Specimens of square cross-section of variable side length $\mathrm{w}(\mathrm{w} \in[4,111] \mathrm{mm})$ were cut from the same PC plate of thickness $4.85 \mathrm{~mm}$. The direction of ultrasound propagation was along the plate thickness. Note that thickness and lateral dimensions are larger than the US wavelength (see Table 1). 
A total of 55 bone specimens were harvested from the left femora of 29 human cadavers. The femurs were provided by the Départment Universitaire d'Anatomie Rockefeller (Lyon, France) through the French program on voluntary corpse donation to science. The tissue donors or their legal guardians provided informed written consent to give their tissue for investigations, in accord with legal clauses stated in the French Code of Public Health. Among the 29 donors, 16 were females and 13 were males $(77.83 \pm 11.39$ years old, mean \pm SD). The fresh material was frozen and stored at $-20^{\circ} \mathrm{C}$.

The specimens were slowly thawed and then, for each femur, approximately a $10 \mathrm{~mm}$ thick cross sectional slab was cut perpendicular to the bone axis at the mid-diaphysis. Then, using a watercooled low-speed diamond wire saw (Model 3241, Well, Lyon, France), two rectangular parallelepiped shaped specimens were prepared in the lateral and medial anatomical quadrants of each cross section. The nominal specimen size was $3 \times 4 \times 5 \mathrm{~mm}^{3}$ in radial (axis 1), circumferential (axis 2) and axial (axis 3 ) directions, respectively, defined by the anatomic shape of the femoral diaphysis. All specimens were kept hydrated during specimen preparation and between measurements.

\subsection{Ultrasonic velocity measurements}

BWV was measured with different pairs of matched contact transducers (Table 1), differing both in central frequency and diameter $\phi$. Transducers were excited with a pulser with $200 \mathrm{MHz}$ (-3 dB) US bandwidth (Panametrics 5900PR). The received signal was digitized and stored using an acquisition card (Agilent Acquiris DP240) and postprocessed with a custom MatLab program (The Mathworks Inc., Natick, MA). For PC specimens, all pairs of transducers were used while for cortical bone, only two pairs were used, namely V105 and V152, for longitudinal and shear waves, respectively. Typical wavelengths in PC associated to each transducer are given in Table 1 . For bone, the choice of the transducers was driven by the dimensions of the specimens and by the scale of interest to measure the bone elasticity. That is, at the chosen frequency $(2.25 \mathrm{MHz}$ and $1 \mathrm{MHz}$, for longitudinal and shear waves, respectively), the resulting wavelength $(\sim 1.7 \mathrm{~mm})$, which defines the probing scale, guaranteed to retrieve the bone mesoscopic elasticity (i.e. at a scale much larger than the vascular pores)(Grimal et al., 2011).

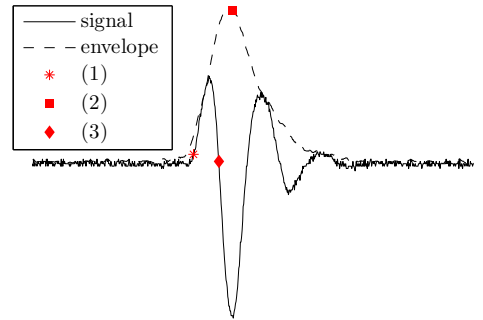

Figure 1: Criteria to define the time-of-flight. (1) first arrival; (2) maximum amplitude of the envelope; (3) first zerocrossing;

The BWV (longitudinal or shear) $v$ was calculated as $v=d / \Delta t$, where $d$ is the propagation distance in the specimen, and $\Delta t$ is a time delay. The latter was calculated as a difference between the time-of-flight (TOF) of the signal propagating through the specimen and that of a reference signal. The propagation distance, $d$, i.e., the specimen's dimension between the transducers, was measured by a digital calliper (precision $\pm 0.01 \mathrm{~mm}$ ). In order to minimize the uncertainty of this measurement, four measurements were averaged. Precisely, the measurement were taken after repositioning the calliper on different location of the opposite surfaces to account for possible parallelism defects.

Four different signal processing techniques were used to define the TOF (Figure 1): (1) the first arrival of the wave defined by a threshold of $5 \%$ of the maximum amplitude of the signal; (2) the maximum amplitude of the envelope defined by the Hilbert transform; (3) the first zero-crossing of the signal; and (4) the phase velocity at the central frequency of the transducer (Droin et al., 1998).

For longitudinal waves, $\Delta t$ was obtained as the difference between the arrival time of the US pulse (determined using one of the proposed signal processing techniques) with the specimen inserted in between the transducers and the arrival time of a reference signal taken with the transducers in contact. Figure 2 shows an example of the longitudinal waveforms in PC and bone and the reference waveform.

For small propagation distances, the shear wave signal generated in the sample by shear wave transducers may not be well separated from a small amplitude spurious longitudinal wave signal also generated by the transducer. This is in general an issue for bone specimens. To circumvent this issue, a polymethyl methacrylate (PMMA) plate of thick- 
Table 1: Ultrasonic transducers

\begin{tabular}{lcccc}
\hline Transducer & Wave Type & $\begin{array}{c}\text { Diameter } \\
\phi[\mathrm{mm}]\end{array}$ & $\begin{array}{c}\text { Central frequency } \\
{[\mathrm{MHz}]}\end{array}$ & $\begin{array}{c}\text { Wavelength PC } \\
{[\mathrm{mm}]}\end{array}$ \\
\hline V105 Panametrics & longitudinal & 19 & 2.25 & 0.94 \\
V110RM Panametrics & longitudinal & 6 & 5 & 0.42 \\
CHG102 NDT systems & longitudinal & 6 & 10 & 0.19 \\
V152 Panametrics & shear & 25 & 1 & 1 \\
V154 Panametrics & shear & 13 & 2.25 & 0.45 \\
SF051 CTS Valpey Corporation & shear & 3 & 5 & 0.22 \\
\hline
\end{tabular}
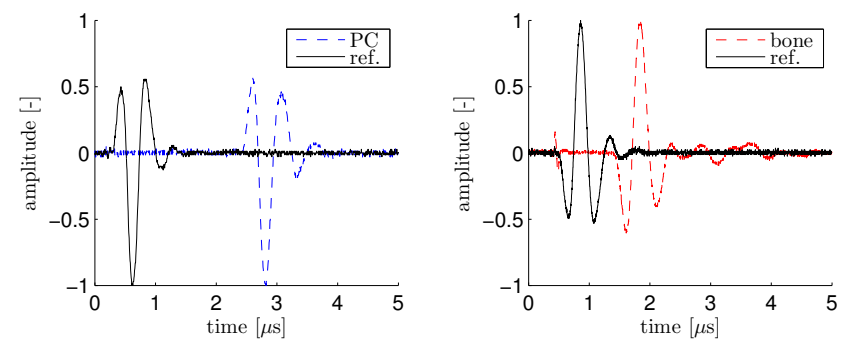

Figure 2: Example of longitudinal wave signals using transducers at 2.25 MHz. The reference signal and signal through the specimen are shown for PC (left) and bone (right).
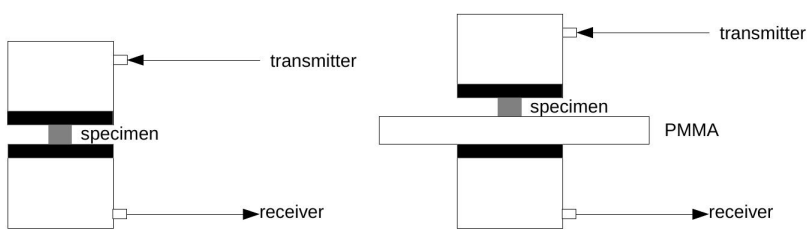

Figure 3: Configuration of transducers for ultrasonic measurements in cortical bone. Longitudinal (left) and shear wave (right) velocity measurements.

ness $9.36 \mathrm{~mm}$ was interposed between the sample and one of the transducers (Figure 3). This increases the traveling distance and allows for a better separation of the longitudinal and shear signals. Accordingly, the reference signal for bone specimens was measured through the PMMA plate. For PC samples, it was not necessary to increase the shear wave traveling distance; accordingly the reference signal was measured with the transducers in contact. Examples of the waveform of shear waves propagation through $\mathrm{PC}$ and bone are shown in Figure 4.

To ensure efficient transmission of the incident waveforms into the specimen, water was used as a couplant for longitudinal waves and honey for the case of shear waves.
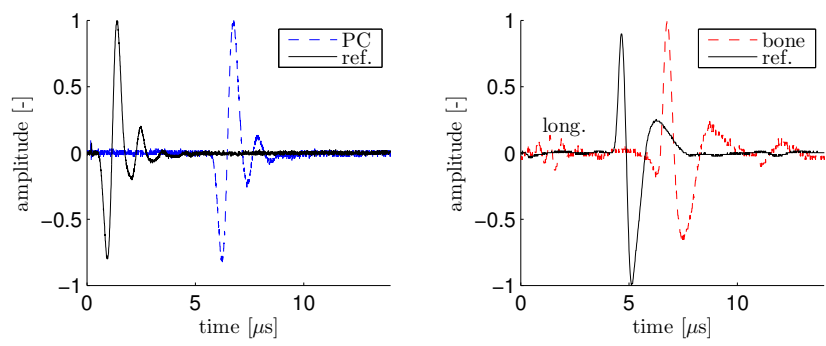

Figure 4: Example of shear wave signals using transducers at $1 \mathrm{MHz}$. The reference signal and signal through the specimen are shown for PC (left) and bone (right). Note the satisfactory splitting of shear and longitudinal signal through bone achieved by interposing a PMMA plate.

Six successive measurements with intermediate repositioning were done for all measurement cases. In subsequent analyses the averaged velocity value determined from the six measurements is used.

In summary, the following measurements were conducted:

- in PC: shear and longitudinal BWV for each size w (11 specimens), for each of the 4 signal processing methods, and with each of the 6 transducer pairs listed in Table 1.

- in bone: shear and longitudinal BWV for each of the 4 signal processing methods with two transducer pairs, V152 and V105, respectively.

\subsection{Resonant Ultrasound Spectroscopy}

For the purpose of comparison, stiffness was evaluated in bone by resonant ultrasound spectroscopy (RUS). This method has been recently introduced to measure cortical bone (Bernard et al., 2013, 2015). In RUS, all elastic constants are determined from a single measurement configuration and the complexity of wave propagation in a finite specimen is taken into account through a model. Thus, RUS is supposed to be free of bias associated with 
specimen size and can provide a more accurate assessment of stiffness, in particular for shear coefficients and small specimens.

The method used was described in detail in (Bernard et al., 2014). Briefly, a bone specimen was held by two opposite corners between two ultrasonic transducers (V154, Panametrics, Waltham, MA), one for emission and one for reception, to achieve a free boundary condition for vibration. The frequency response of the vibration in the frequency range $100-500 \mathrm{kHz}$, tuned so as to measure the 30-40 first resonant frequencies, was recorded by a vector network analyzer (Bode 100, Omicron Electronics GmbH, Klaus, Austria) and a broadband charge amplifier (HQA-15 M-10 T, Femto Messtechnik GmbH, Berlin, Germany). Six consecutive measurements were performed on each specimen. Between each measurement, the specimen was turned with a slightly different orientation in order to maximize the number of detectable resonant frequencies. Then, the resonant frequencies of the specimen were extracted from the six measured responses using the method dedicated to high damping material (Lebedev, 2002). Finally, knowing the apparent mass and dimensions of each specimen, the elastic coefficients were automatically calculated by solving the inverse problem formulated in a Bayesian framework (Bernard et al., 2015).

\subsection{Data analysis}

The precision error of the measurements was quantified in PC by calculating the standard deviation (SD) of the BWV obtained from 6 measurements. To analyze the influence of the signal processing method, specimen size (w), and transducer choice, all BWV measured in PC specimens were analyzed with three-way analysis of variance (ANOVA). Also, in order to further investigate the influence of specimen size, stiffness and precision errors were analyzed in $\mathrm{PC}$ with respect to the ratio specimen size-transducer diameter, $\mathrm{w} / \phi$, and using linear regressions. To analyze the influence of the signal processing method in bone, BWV were analyzed with one-way ANOVA. Post hoc Tukey-Kramer tests were used to compare individual groups when appropriated. Determined stiffness values with RUS and BWV methods were compared using ANOVA and post hoc TukeyKramer tests. Statistical analysis was carried out using Matlab Statistics Toolbox Release 2014a (The MathWorks Inc., Natick, Massachusetts, United
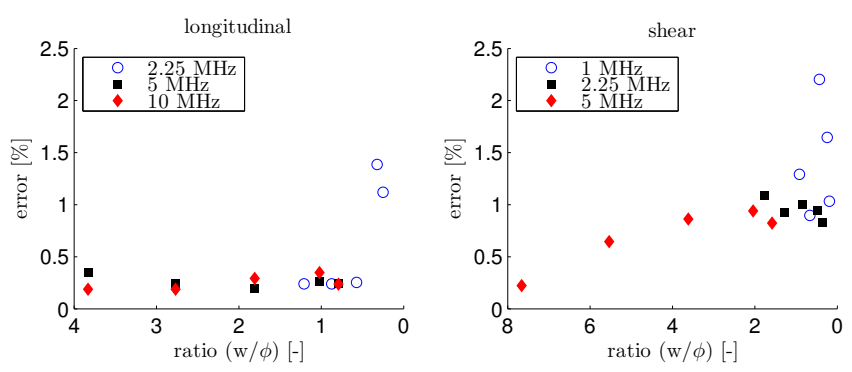

Figure 5: Evolution of precision error of stiffness in PC specimens with transverse dimension smaller than $20 \mathrm{~mm}$ $(\mathrm{w} \leq 20)$ at different excitation frequencies and using the first arrival wave criterion. Values obtained using different transducers are plotted as a function of $w / \phi$. Left: longitudinal stiffness $C_{11}$; right: shear stiffness $C_{44}$.

States). Data were considered statistically significant for $p<0.05$.

\section{Results}

Overall, precision error decreased when frequency increased. The averaged error using the different transducers (at different central frequencies) ranged from $0.24 \%$ to $0.85 \%$ and from $0.57 \%$ to $1.24 \%$ for longitudinal and shear stiffness, respectively. Figure 5 shows the precision error calculated in PC specimens with transverse dimension $\mathrm{w}$ smaller than $20 \mathrm{~mm}$ and using the first arrival of the wave to define the TOF. Similar results were found for the rest of the signal processing techniques. In general, the velocity defined by the maximum amplitude of the envelope led to the largest precision errors and first-zero crossing and first arrival of the wave criteria to the smaller ones. This error increased at smaller specimen size for shear stiffness but was more constant for longitudinal stiffness (no significantly high correlation in any longitudinal case).

The distribution in form of box-plots of the elastic coefficients measured in all the PC specimens (including all specimen sizes) and grouped by frequency and signal processing technique are depicted in Figure 6. Three-way ANOVA revealed differences of both longitudinal and shear stiffness associated to transducer choice and signal processing method $\left(p<10^{-4}\right)$. Interestingly, PC stiffness calculated using the arrival time criterion was consistently higher except for the longitudinal BWV at $10 \mathrm{MHz}$. Post hoc Tukey tests showed that measurements with the different transducers were all different except for longitudinal stiffness at $5 \mathrm{MHz}$ and $10 \mathrm{MHz}$, which did not show any significant 

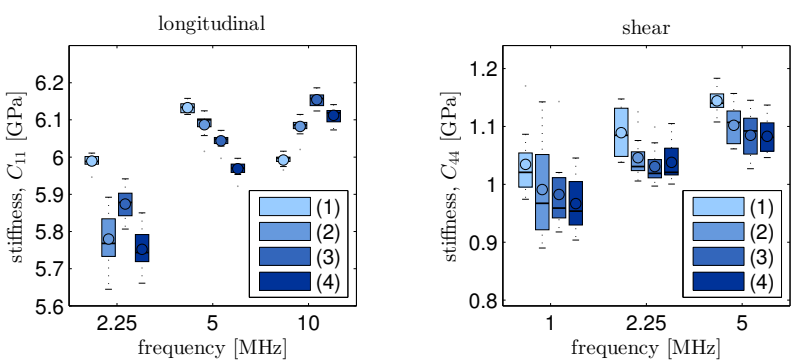

Figure 6: Longitudinal $\left(C_{11}\right.$, left $)$ and shear $\left(C_{44}\right.$, right $)$ stiffness measured in polycarbonate specimens at different excitation frequencies and using 4 different signal processing methods to calculate the US velocity. (1) first arrival; (2) maximum amplitude of the envelope; (3) first zero-crossing. (4) phase velocity. Median (lines within boxes), mean (circles within boxes), interquartile (boxes) and extreme values (whiskers) are shown.
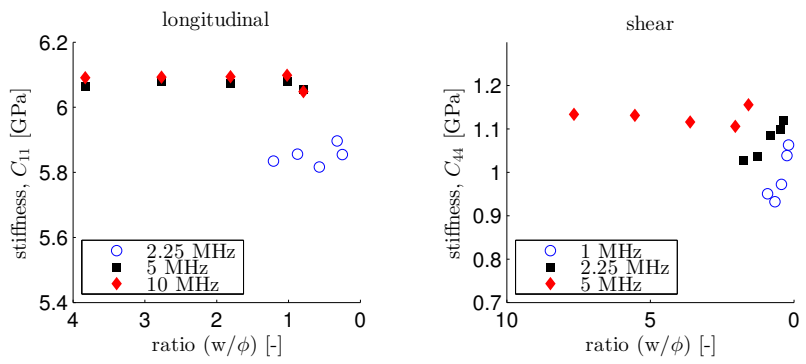

Figure 7: Measured stiffness in polycarbonate specimens with transverse dimension smaller than $20 \mathrm{~mm}(\mathrm{w} \leq 20)$ at different excitation frequencies. Left: longitudinal stiffness $C_{11}$; right: shear stiffness $C_{44}$.

difference. In general, stiffness increased at higher frequencies, which is consistent with the expected behavior for a viscoelastic solid (Lakes, 2009).

We found significant differences associated with specimen's dimension in shear stiffness $C_{44}$ (expressed as w $/ \phi$ ) (Figure 7) but not in longitudinal stiffness $C_{11}$ (ANOVA: $p<10^{-4}, p=0.17$ for shear and longitudinal stiffness, respectively). Shear stiffness was correlated with the aspect ratio $\mathrm{w} / \phi$ and this correlation increased for aspect ratios smaller than two $\left(R^{2}>0.4, p<0.05\right.$ in all cases $)$. Longitudinal stiffness was mostly constant throughout the whole range of specimen dimensions and neither strong nor significant correlation was found ( $R^{2} \leq 0.189, p>0.18$ in all cases).

Dependency of stiffness on the signal processing technique was also found in cortical bone $\left(p<10^{-4}\right.$, ANOVA). Post hoc Tukey tests indicated that for longitudinal waves, the phase velocity gave the lowest stiffness values, while velocity defined by the arrival criterion yielded the highest values. In the
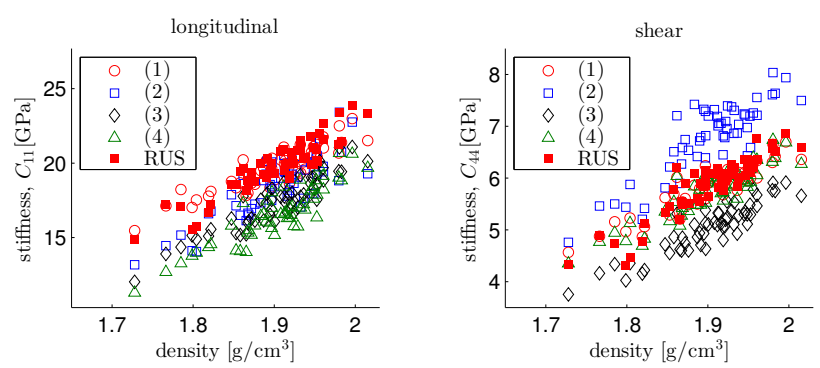

Figure 8: Comparison of stiffness coefficients calculated by different signal processing methods and RUS in cortical bone. (1) first arrival; (2) maximum amplitude of the envelope; (3) first zero-crossing; (4) phase velocity. Left: longitudinal stiffness $C_{11}$; right: shear stiffness $C_{44}$.

case of shear waves, the highest stiffness values corresponded to the velocity defined by the maximum amplitude of the envelope and the lowest ones to the velocity defined by the first-zero crossing of the signal. The measured stiffness coefficients calculated by using different signal processing methods are summarized in Table 2 and compared to those measured by RUS. Because we expect a variation of stiffness with mass density (Bernard et al., 2015), the shear and longitudinal stiffness values obtained from BWV and RUS are plotted as a function of mass density in Figure 8. Note that only $C_{11}$ and $C_{44}$ are shown but similar results were found for the other coefficients.

Longitudinal stiffness coefficients $\left(C_{11}, C_{33}\right)$ obtained from RUS did not significantly differ from those obtained from BWV using the arrival criterion. Shear stiffness coefficients $\left(C_{44}, C_{66}\right)$ obtained from RUS did not significantly differ from those obtained from BWV using either of the arrival time or phase velocity criteria (Table 2).

\section{Discussion}

Previous studies have shown that results obtained in terms of US wave velocity are dependent on the method used to estimate the speed of sound in trabecular bone due to the frequency dependence of the attenuation coefficient and the velocity dispersion of trabecular bone (Haiat et al., 2006; Nicholson et al., 1996). Cortical bone is also a dissipative medium, but a lesser extent compared to cancelous bone (Laugier and Haïat, 2011; Trebacz and Gawda, 2001) and the influence of the method to estimate the US wave velocity has not been reported yet as far as we know. We have shown that, 
Table 2: Average stiffness coefficients (SD) of cortical bone obtained with RUS and BWV method using different signal processing: (1) first arrival; (2) maximum amplitude of the envelope; (3) first zero-crossing; (4) phase velocity.

\begin{tabular}{lccccc}
\hline & \multicolumn{4}{c}{ Signal processing method } & \multirow{2}{*}{ RUS } \\
\cline { 2 - 5 }$(\mathrm{GPa})$ & $(1)$ & $(2)$ & $(3)$ & $(4)$ & \\
\hline$C_{11}$ & $19.47(1.72)$ & $18.17(2.45)$ & $17.11(2.17)$ & $16.37(2.35)$ & $19.35(2.94)$ \\
$C_{33}$ & $27.72(1.77)$ & $26.59(2.15)$ & $26.03(2.06)$ & $25.30(2.27)$ & $28.33(3.68)$ \\
$C_{44}$ & $5.74(0.55)$ & $6.66(0.93)$ & $4.97(0.61)$ & $5.65(0.67)$ & $5.75(0.78)$ \\
$C_{66}$ & $4.35(0.60)$ & $4.70(0.85)$ & $3.79(0.66)$ & $4.23(0.69)$ & $4.28(0.78)$ \\
\hline
\end{tabular}

in cortical bone and $\mathrm{PC}$, which intrinsic attenuation is close to that of bone, the signal processing method used to define the velocity can lead to significantly different stiffness estimates. These differences, introduced by the signal processing method, represent approximately $1.5 \%$ and $2.7 \%$ for longitudinal and shear stiffness in the case of PC, and increases up to $5.7 \%$ and $10.4 \%$ in the case of longitudinal and shear stiffness of cortical bone, respectively. It is noteworthy that the measurement of shear stiffness is more sensitive to the measurement conditions than longitudinal stiffness. Our results point out the possible effects of dispersion in cortical bone, which result is to distort signals and leads to different values of BWV using different signal processing methods.

Signal distortion can also be the result of interferences within the specimens due to reflection of bulk waves within the specimen and near-field diffraction effects. We have observed that specimen's transverse dimension has an effect on shear stiffness coefficients. Similar effects of the transverse specimen dimension on velocity were reported previously in polymers, but correlations were weaker than the ones found here (Kohles et al., 1997). They attributed this size effect mainly to the transition from bar to bulk wave propagation. The present study however, in which all specimen dimensions are larger than the wavelength, suggests that there may be other explanations besides the bar-bulk wave transition for the observed effects on transverse stiffness.

The feasibility of BWV measurement to retrieve stiffness coefficients in small specimens such the ones used in cortical bone application (few millimeters) has already received attention in the literature (Kohles et al., 1997; Schwartz-Dabney and Dechow, 2002). It has been shown that measurement repeatability (precision) improves with an increase in the wave transmission distance (propagating dimension). Uncertainty in the determination of the length of wave path may be responsible, in part, for the precision dependency on the propagation dimension. The uncertainty on the determination of the dimensions is affected by both the quality of the cuboid shape (parallelism error) and the precision of the calliper. The specimen dimension is measured at four locations after repositioning. These four values are averaged, compensating to a certain extent for the uncertainty due to parallelism error. Nevertheless, the uncertainty of dimensions induced by measurements, e.g., measured by a digital calliper, may impact stiffness calculations. In this study, considering a dimension uncertainty of $\pm 0.01 \mathrm{~mm}$, defined by the calliper precision, the uncertainty error of the calculated stiffness can be up to $0.41 \%$ in $\mathrm{PC}$ and $0.67 \%$ in bone when the wave propagation is along the radial dimension $\left(C_{11}, C_{55}\right.$ and $\left.C_{66}\right)$, which is the shortest one $(3 \mathrm{~mm})$. Note that these values should be considered as upper bounds of the error since in the actual protocol, the dimension of a specimen is taken to be the average of four measurements. Overall, the error due to dimension uncertainty may be comparable to the one introduced by frequency while main differences in elasticity are related to the signal processing technique. Our study also points out that not only the propagation dimension but also the transverse one determines the precision of the measurements. In particular, the increased variability of transverse stiffness measurements with smaller specimen size was of greater concern than in the case of longitudinal stiffness.

There are different possible explanations for these differences between shear and longitudinal waves. One important reason may be the greater difficulty in measuring small specimens, which in the case of a shear waves transducer, there are extra difficulties arising from the transducer polarization, the delicate positioning and coupling with the specimen (Maynard, 1992; Grimal et al., 2009). In addition, in the case of small specimens, additional uncertain- 
ties may arise from the relative position between specimen and transducer. On the other hand, a conventional US transducer always generates both longitudinal and shear waves that not always can be correctly separated (Tang et al., 1994). It is possible that in the small specimens $(\mathrm{w} / \phi \leq 2)$ this phenomenon may have more importance. These additional difficulties may increase the measurement error.

Finally, although this study sheds light on some problems for measuring elastic properties in cortical bone, some others remain. As has been shown here, increasing the frequency improves the precision of the measurements. However, scaling up the frequency is limited by the scale of interest and the wavelength must remain much larger than bone heterogeneities. At the mesoscale, this length is limited to the representative volume element (RVE), which is approximately $1.5 \mathrm{~mm}$ (Grimal et al., 2011). Moreover, the attenuation of ultrasound waves increases with frequency, particularly for transverse waves. Motivated to overcome these difficulties, RUS has been recently adapted to bone (Bernard et al., 2013), which has shown better precision compared to ultrasound velocity measurements. There is in principle no limitation in size (as long as the specimen is larger than the RVE), since all the complexity of wave propagation in a finite specimen is taken into account instead of assuming propagation of pure bulk waves. In the present study we have shown that, for the particular measurement conditions in cortical bone, stiffness obtained from RUS was not significantly different from the stiffness obtained from the signal velocity based on the first arrival of the signal.

This study has several limitations. We did not determine the accuracy (trueness) of the BWV or stiffness values because we lack reference values. Elasticity measurement with RUS has been validated previously by comparing the derived elastic coefficients with reference values of Young's modulus and Poisson ratio of synthetic bone samples (Bernard et al., 2014). This validation was however limited to two samples and the variation of the elastic properties with frequency was not further investigated. Additional RUS validation measurements are necessary before RUS can be used as a reference to discuss small inaccuracies of bone elasticity measurements with BWV. Also, the effect of varying the measurement frequency was not investigated in bone. Although we could quantify the effect of the frequency in PC (a small percent- age of stiffness) care should be taken to extrapolate this result to bone. Indeed, bone is not only viscoelastic like PC but is also heterogeneous and at frequencies in the range $5-10 \mathrm{MHz}$ and higher the wavelength may not be sufficiently large compared to the RVE to probe effective elastic properties. Finally, we could not provide an unambiguous explanation for the observed effect of transducer size on measured shear stiffness. This was prevented by the complexity of the mechanism of wave excitation. This would deserve further analysis using, e.g. numerical simulations.

There is no standard protocol to measure cortical bone stiffness coefficients based on BWV and we have evidenced that the measurement method can have an effect on the stiffness values. For smooth progress in bone research, it would be beneficial to comply a well defined protocol when measuring bone stiffness coefficients based on BWV. Such a protocol must be defined according to several conditions (specimen size, frequency and signal processing). Although those settings taken together are difficult to reconcile, in practice, a compromise must be done. Based on the results of the present study, we may formulate the following guidelines. The central frequency of shear and longitudinal wave transducers should be chosen in order to achieve a similar wavelength in bone, typically larger than $1.5 \mathrm{~mm}$. $2.25 \mathrm{MHz}$ and $1 \mathrm{MHz}$ for longitudinal and shear waves, respectively, is a popular choice. Since the signal processing method has a significant effect on the stiffness, it should be clearly reported in all studies. We recommend that the beginning of the signal be measured for the TOF estimation. This marker is in general less sensitive to signal dispersion and its precision is reasonable. Furthermore, this criterion leads to the same stiffness values as the values calculated by RUS. Finally, to guarantee a correct separation between shear and longitudinal components, the interposition of a plate of PMMA or PC, with impedance relatively close to that of bone, was found to be efficient to measure the TOF of shear waves. Unfortunately, the specimen size cannot be standardized because this depends on the available material; nevertheless, the recommendation is to extract the largest cuboid specimen possible in the cortical bone thickness avoiding large pores and excessive porosity gradient in the radial direction. 


\section{Conclusion}

There is a need for standardizing the ultrasonic bulk wave velocity measurements in cortical bone. Effects of the signal processing method, specimen size and frequency on the resulting stiffness have been experimentally investigated. Results indicate that (1) there is a relatively increased measurement error at lower frequencies; (2) the error increases in shear stiffness as transverse section decreases; and (3) resulting stiffness significantly depends on the signal processing technique used to estimate the velocity. For human cortical bone, over all the different analysed velocities, the one calculated from the first arrival of the signal leads to similar results to the resonant ultrasound spectroscopy method.

\section{Acknowledgment}

The authors would like to thank Rémy Gauthier, Hélèn Follet and David Mitton for the collection of bone specimens. This work has received financial support from the Agency National Research under the ANR-13-BS09-0006 MULTIPS and ANR14-CE35-0030-01 TaCo-Sound projects.

\section{References}

Ashman, R., Cowin, S., Van Buskirk, W., Rice, J., 1984. A continuous wave technique for the measurement of the elastic properties of cortical bone. Journal of biomechanics 17 (5), 349-361.

Auld, B. A., 1973. Acoustic fields and waves in solids. New York: Wileyand, Sons. p. 218.

Baumann, A. P., Deuerling, J. M., Rudy, D. J., Niebur, G. L., Roeder, R. K., 2012. The relative influence of apatite crystal orientations and intracortical porosity on the elastic anisotropy of human cortical bone. Journal of biomechanics 45 (16), 2743-2749.

Bernard, S., Grimal, Q., Laugier, P., 2013. Accurate measurement of cortical bone elasticity tensor with resonant ultrasound spectroscopy. Journal of the mechanical behavior of biomedical materials 18, 12-19.

Bernard, S., Grimal, Q., Laugier, P., 2014. Resonant ultrasound spectroscopy for viscoelastic characterization of anisotropic attenuative solid materials. The Journal of the Acoustical Society of America 135 (5), 2601-2613.

Bernard, S., Marrelec, G., Laugier, P., Grimal, Q., 2015. Bayesian normal modes identification and estimation of elastic coefficients in resonant ultrasound spectroscopy. Inverse Problems 31 (6), 065010.

Droin, P., Berger, G., Laugier, P., 1998. Velocity dispersion of acoustic waves in cancellous bone. IEEE transactions on ultrasonics, ferroelectrics, and frequency control 45 (3), 581-592.

Engelke, K., van Rietbergen, B., Zysset, P., 2016. Fea to measure bone strength: A review. Clinical reviews in bone and mineral metabolism 14 (1), 26-37.
Granke, M., Grimal, Q., Saïed, A., Nauleau, P., Peyrin, F., Laugier, P., 2011. Change in porosity is the major determinant of the variation of cortical bone elasticity at the millimeter scale in aged women. Bone 49 (5), 1020 - 1026.

Grimal, Q., Haupert, S., Mitton, D., Vastel, L., Laugier, P., 2009. Assessment of cortical bone elasticity and strength: mechanical testing and ultrasound provide complementary data. Medical engineering \& physics 31 (9), 11401147.

Grimal, Q., Raum, K., Gerisch, A., Laugier, P., 2011. A determination of the minimum sizes of representative volume elements for the prediction of cortical bone elastic properties. Biomechanics and modeling in mechanobiology $10(6), 925-937$.

Haiat, G., Padilla, F., Cleveland, R. O., Laugier, P., 2006. Effects of frequency-dependent attenuation and velocity dispersion on in vitro ultrasound velocity measurements in intact human femur specimens. ieee transactions on ultrasonics, ferroelectrics, and frequency control 53 (1), 39-51.

Kohles, S., Bowers, J., Vailas, A., Vanderby, R., 1997. Ultrasonic wave velocity measurement in small polymeric and cortical bone specimens. Journal of biomechanical engineering 119 (3), 232-236.

Lakes, R., Yoon, H. S., Katz, J. L., 1986. Ultrasonic wave propagation and attenuation in wet bone. Journal of biomedical engineering 8 (2), 143-148.

Lakes, R. S., 2009. Viscoelastic materials. Cambridge University Press. p. 63-65.

Lang, S. B., 1969. Elastic coefficients of animal bone. Science 165 (3890), 287-288.

Laugier, P., Haïat, G., 2011. Bone quantitative ultrasound. Vol. 576. Springer. p. 1-28.

Lebedev, A. V., 2002. Method of linear prediction in the ultrasonic spectroscopy of rock. Acoust. Phys. 48, 339346.

Lefèvre, E., Lasaygues, P., Baron, C., Payan, C., Launay, F., Follet, H., Pithioux, M., 2015. Analyzing the anisotropic hooke s law for children s cortical bone. Journal of the mechanical behavior of biomedical materials 49, 370-377.

Maynard, J. D., 1992. The use of piezoelectric film and ultrasound resonance to determine the complete elastic tensor in one measurement. The Journal of the Acoustical Society of America 91 (3), 1754-1762.

Nicholson, P., Lowet, G., Langton, C., Dequeker, J., Van der Perre, G., 1996. A comparison of time-domain and frequency-domain approaches to ultrasonic velocity measurement in trabecular bone. Physics in medicine and biology 41 (11), 2421.

Njeh, C., Hodgskinson, R., Currey, J., Langton, C., 1996. Orthogonal relationships between ultrasonic velocity and material properties of bovine cancellous bone. Medical engineering \& physics 18 (5), 373-381.

Orías, A. A. E., Deuerling, J. M., Landrigan, M. D., Renaud, J. E., Roeder, R. K., 2009. Anatomic variation in the elastic anisotropy of cortical bone tissue in the human femur. Journal of the mechanical behavior of biomedical materials 2 (3), 255-263.

Phillips, D., North, A., Pethrick, R., 1977. Ultrasonic studies of polycarbonate, polysulfone, and polyether sulfone. Journal of Applied Polymer Science 21 (7), 1859-1867.

Rho, J.-Y., 1996. An ultrasonic method for measuring the elastic properties of human tibial cortical and cancellous bone. Ultrasonics 34 (8), 777-783.

Rudy, D. J., Deuerling, J. M., Orías, A. A. E., Roeder, R. K., 
2011. Anatomic variation in the elastic inhomogeneity and anisotropy of human femoral cortical bone tissue is consistent across multiple donors. Journal of biomechanics 44 (9), 1817-1820.

Schwartz-Dabney, C., Dechow, P., 2002. Accuracy of elastic property measurement in mandibular cortical bone is improved by using cylindrical specimens. Journal of biomechanical engineering 124 (6), 714-723.

Tang, X., Zhu, Z., Toksöz, M., 1994. Radiation patterns of compressional and shear transducers at the surface of an elastic half-space. The Journal of the Acoustical Society of America 95 (1), 71-76.

Trebacz, H., Gawda, H., 2001. The estimation of structural anisotropy of trabecular and cortical bone tissues based on ultrasonic velocity and attenuation. Acta of Bioengineering and Biomechanics 3 (2), 41-48.

van Rietbergen, B., Ito, K., 2015. A survey of micro-finite element analysis for clinical assessment of bone strength: the first decade. Journal of biomechanics 48 (5), 832-841. 